\title{
Integration Design of Temperature Sensor and Double RFID Tag
}

\author{
Xiangdong Zhang, Changming Li, Xiaoqiu Gao and Lijie Li ${ }^{*}$
}

College of Business Administration, Hebei Normal University of Science and Technology, Qinhuangdao, China, 066004

\begin{abstract}
A new design about the integration of temperature sensor and RFID tag is provided. This is a dual-tag design, in which the temperature element is connected to one of the RFID tag antennas to form a parallel structure. In this design, some of the functions of the sensor are transferred to the RFID reader so that some components of the sensor can be cut, reducing the production costs of existing design. By defining the signal strength of the first tag as a reference value, the problem of setting a standard is solved in the temperature detection process. RF module can be used to make the energy transformation without requiring the entire design to have an external continuous power source to provide energy. In addition, experimental data proves the feasibility of this design.
\end{abstract}

Keywords: Dual-tag, radio frequency identification, sensor, temperature sensitive component.

\section{INTRODUCTION}

RFID is a main component of the internet of things. The internet of things have three main characters which are 'sense', ' transmission' and 'intelligence'. The integration of RFID tag and sensor facility is an exhibition of these characters. As to the integration of sensor and RFID tag, Weifeng Liu and Yiqi Zhuang adopted a dual-oscillation instrument structure, which made sampling and counting by using signals occurred in the course of standard clock influenced by temperature, and got measure value of temperature according to the linear relation between courting value and temperature [1]. As to the link between sensor and a certain module inside RFID tag, Lei Li, Sheng Xie and Xiaozong Huang transferred the temperature information into digital signals by using switch circuit. Those signals were used by control module of RFID tag [2]. Haibin Meng and Hongyu Zhang linked the circuit to check and measure temperature and humidity and CMOS chip [3]. The check and measure results were transmitted to reading instrument via radio module by antenna in the operation of control module. As to the link between sensor and multi-module inside RFID tag, Bin Yang linked control module to sensor, radio frequency module and store module separately [4]. The control module could receive dictates from radio frequency module, and turn on the sensor to measure temperature, then store the register data of measured temperature into memorizer. Lingrong Kong et al linked temperature sensor to control module through command and data. Then control module was linked to radio frequency module [5]. This set of combination could measure and register the change of working environment temperature and read out the data stored inside tag $[6,7]$. About the problem of power supply in integration of sensor and RFID

\footnotetext{
*Address correspondence to this author at the College of Business Administration, Hebei Normal University of Science and Technology, Qinhuangdao, China,066004; Tel: 13930375156;

E-mails: zhangxd452@163.com, hbqhdlcm@163.com
}

tag, Zhigang Hou input the temperature value proceed by processor into store module of RFID tag, then reading instrument read the temperature information inside tag by antenna [8]. In the working process, though passive RFID tag, temperature sensor and processing module need electricity power to work.

Temperature sensor as a most important kind of Sensor instruments is widely used in industry, agriculture, science research, living and other domains, also its application amount is in the first place among most kind of sensors. There is a great application vision in the combination of temperature sensor and RFID technology. This paper firstly introduce the integration of temperature sensor and RFID technology in detail. Then make the design idea clear and propose a new integration design based on analysis of presented research and technology. Finally, the design is testified feasible by experiment.

\section{ANALYSIS OF INTEGRATION BETWEEN TEM- PERATURE SENSOR AND RFID TAG}

Many problems should be considered when integrate temperature sensor and RFID tag, such as cost problems, mark deciding problems in measurement, problems of supply power to devices, problems of choosing integration point position and problems of innovation of integration mode and so on. Reference [9-11] have been a well done in several aspects of these problems.

\subsection{Probability Analysis Of Temperature Sensor Inte- gration}

As for temperature sensor, it is made up of temperature sensitive component, transform component, signal adjusting and transforming circuit and assistant electrical power. Among them, temperature sensitive component plays the core function [12]. Temperature sensitive component could be classified two kinds of caloric resistance and thermocouple. Caloric resistance is made up by using the conductor 


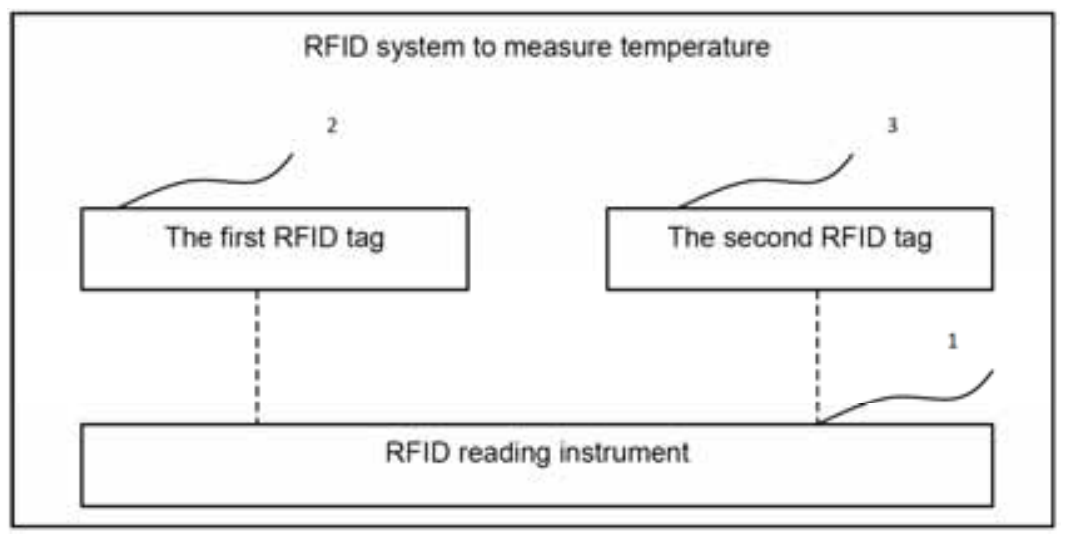

Fig. (1). A dual-label structure to measure temperature.

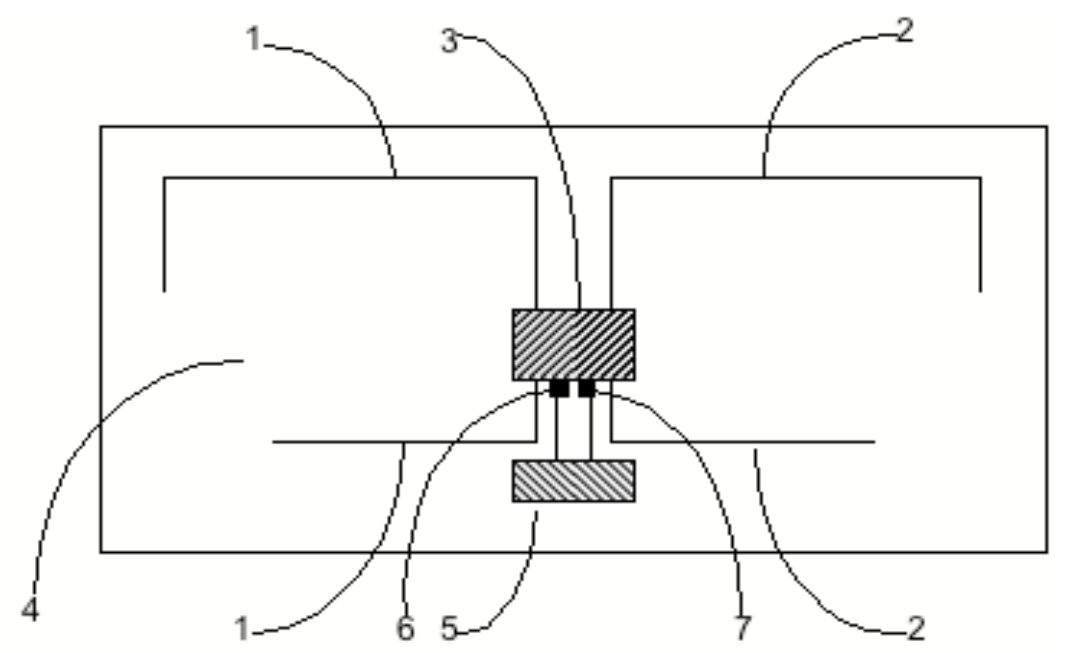

Fig. (2). Sketch figure of the second tag structure.

characteristic that resistance value of conductor change with temperature change. Now the most material used in Caloric resistance are platinum, copper, nickel, manganese and rhodium etc [13]. The design of this paper aims at caloric resistance, a kind of temperature sensor that their output resistance change with temperature. Grasping the essence of electricity signal change in resistance type temperature sensor is a base to realize the integration of temperature sensor and RFID tag.

\subsection{Probability Analysis Of RFID Tag Integration}

Further analysis to RFID technology system indicates that the information interaction of RFID technology system and outside is realized by electronic tag as window. The tag contains CMOS chip module, antenna and card radix, and CMOS chip contains radio frequency module, control module and memorizer module [14]. It can be known that there have four kind of probabilities in integration point position of the two technologies by integrating thoughts of presented studies about integration of sense technology and tag. The four kind of probabilities are as follows: link to antenna, link to radio frequency module, link with control module and link to memorizer module. In addition, linking to multiple modules simultaneously for further extent amalgamation is also one of an ideal technology designs. One point must be specially proposed is character frequency of antenna and signal intensity could be influenced by antenna itself and things linked to it [15]. This is a key point of the whole technology integration thought. To go ahead from this point, we could link temperature sensitive component to antenna of electronic tag, and identify the change of environment temperature by antenna signal frequency or change of intensity.

\section{DUAL-LABEL DESIGN OF TEMPERATURE SEN- SITIVE COMPONENT AND RFID TAG INTEGRA- TION}

By Summarizing the above analysis of temperature sensitive component and RFID tag integration and borrowing the though of reference [16-19], an integration design of them is reached as follows:

A RFID dual-label structure to measure the temperature is depicted as Fig. (1). The design contains RFID reading instrument 1, the first RFID tag 2 and the second RFID tag 3. The first RFID tag 2 and the second RFID tag 3 mentioned above have same CMOS chip and antenna. The second RFID tag 3 mentioned above is a RFID tag with temperature sensitive component, which link to the antenna of the second RFID tag 3 mentioned above.

The structure of the second tag mention above is depicted as Fig. (2). Fig. (2) is a sketch figure of device that links temperature sensitive component and RFID CMOS chip to- 


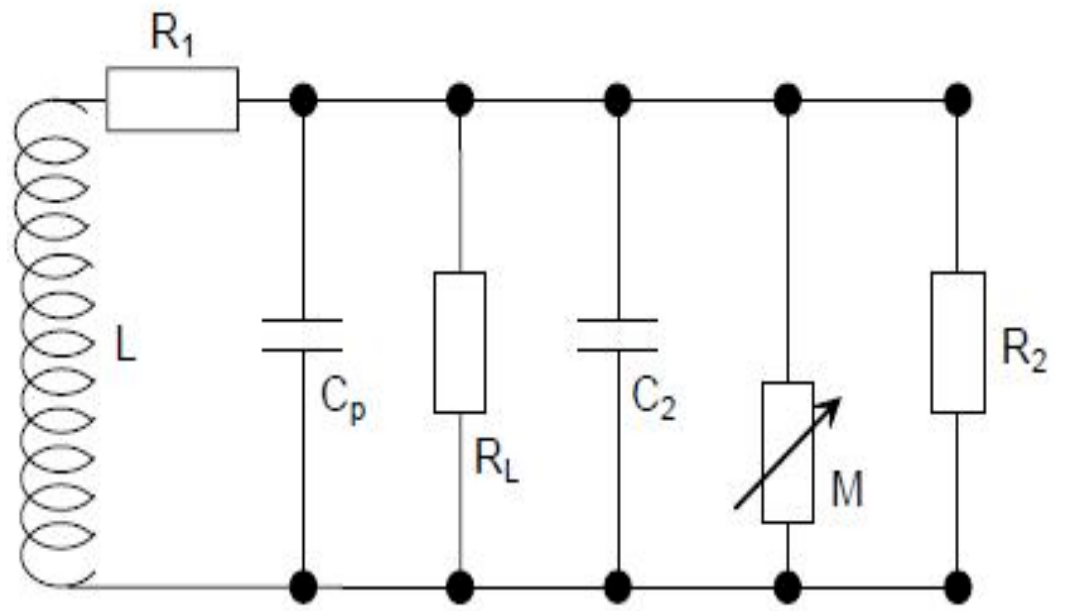

Fig. (3). Equivalent circuit figure of linkage of temperature sensitive component and the second RFID tag.

gether. The device contains a base 4 , a integrate circuit board 3 , a temperature sensitive component 4 , two lead pins 6 and 7, and a double dipole antenna 1 and 2. The two lead pins 6 and 7 are set on the integrate circuit board 3, and temperature sensitive component link to antenna inside CMOS chip in parallel connection structure, and also have same CMOS chip and antenna with the first tag corresponding to the second tag. The temperature sensitive component mentioned above is resistance type temperature sensitive component.

As shown in Fig. (3), it is a sketch figure of linkage of temperature sensitive component and the second RFID tag equivalent circuit. It contains loop inductance (L), autoecious capacitance $\left(\mathrm{C}_{\mathrm{p}}\right)$ and parallel connection capacitance $\left(\mathrm{C}_{2}\right)$, its resonance frequency is:

$f=\frac{1}{2 \pi \sqrt{L^{*} C}}$

Where $\mathrm{C}$ in above formula is parallel connection capacitance of $\mathrm{C}_{\mathrm{p}}$ and $\mathrm{C}_{2}, \mathrm{R}_{1}$ and $\mathrm{R}_{2}$ are equivalent resistance of inductance loop inside circuit and other devices. $\mathrm{M}$ denotes the changeable resistance of temperature sensitive components.

The AC input voltage supply is initially rectified and filtered by a capacitor to a stable DC voltage. In any sudden undesired input variation this capacitor will act as stabilizer and will keep the DC voltage constant to the desired levels. The rectifier output even if a filter is used has a ripple. This DC output is directed to a high frequency chopping system. The output voltage is stable and is regulated by the width of pulses driving the semiconductor power elements. So, the inverter gets as input the power network voltage and manages to produce a stable with no variations AC voltage.

The microcontroller is sampling the input voltage and manages the width of the driving transistor pulses in order to achieve a stable output voltage.

Another example to be discussed is a design with two lead pins and two antenna (double dipole antenna) on the CMOS chip of the second tag. there are two instances in this condition. The first instance is linking temperature sensitive component to the two lead pins, and forming parallel connection structure with the first antenna. In this instance, the first antenna is working under the second resonance frequency, and the second antenna is working under the first resonance frequency. The second instance is linking temperature sensitive component to the two lead pins, and forming parallel connection structure with both antenna. In this instance, both antennas are working under the second resonance frequency.

In the dual-label design scheme, the first tag exits only as an object of reference, while design of the second tag is a key point in the whole structure. As for the structure of the second tag, the structure introduced above is only a 'double lead pin-double antenna' structure, furthermore there still have 'double lead pin-single antenna' structure, 'single lead pin-double antenna' structure, and 'non-lead pin' structure. The 'double lead pin-single antenna' structure refers to there is only one antenna on the second tag, and two lead pins on the CMOS chip of tag. Temperature sensitive components link to this antenna through the two lead pins, and the first tag as an object of reference must be consistent with the second one in structure. The 'single lead pin-double antenna' structure is depicted in Fig. (4), there is only one lead pin 6 on the CMOS chip of tag. this lead pin link outside to one end of temperature sensitive components, and the other end of temperature sensitive components is directly linked to antenna.

It is similar to two lead pins condition when lead pins linkage point inside the CMOS chip, also needs to form a parallel connection structure with antenna of tag. there still have two instances in this conditions. The first instance is to link temperature sensitive components to one lead pin in one end, and the other end link to the first antenna in a parallel connection structure. In this instance, the first antenna is working under the second resonance frequency, and the second antenna is working under the first resonance frequency. The second instance is to link temperature sensitive components to one lead pin in one end, and the other end link to antenna and forming parallel connection structure with both antenna. In this instance, both antennas are working under the second resonance frequency.

'Non lead pin' structure is depicted as Fig. (5), the two end of temperature sensitive components link directly to antenna when there is no lead pin on the CMOS chip of tag. in 


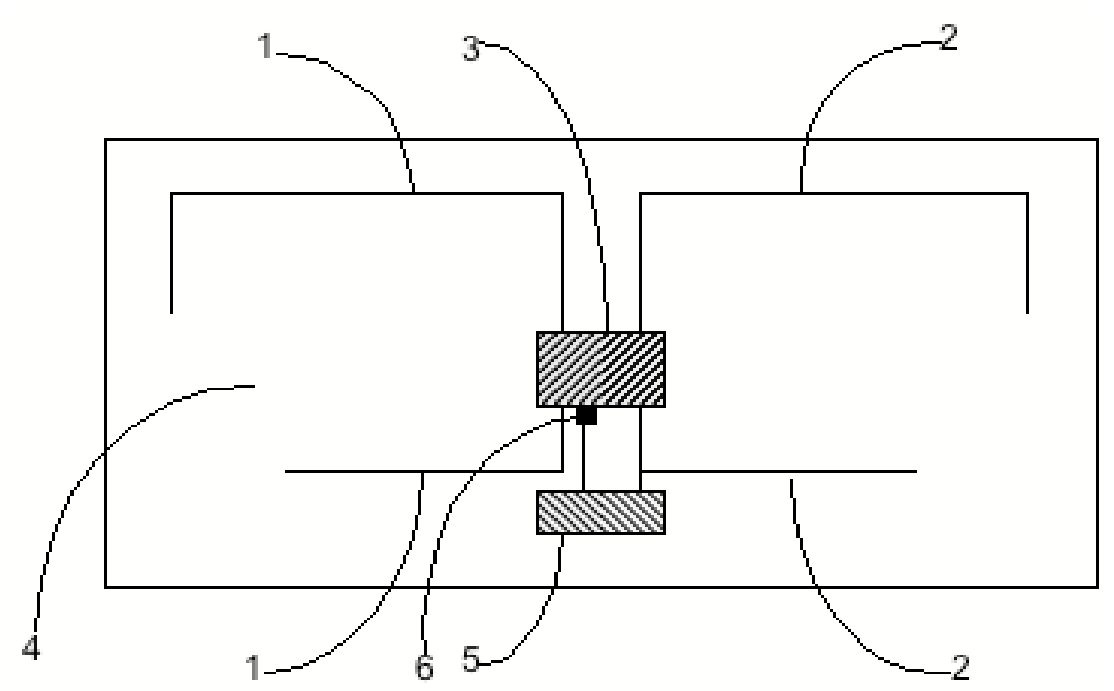

Fig. (4). 'Single lead pin-double antenna' structure.

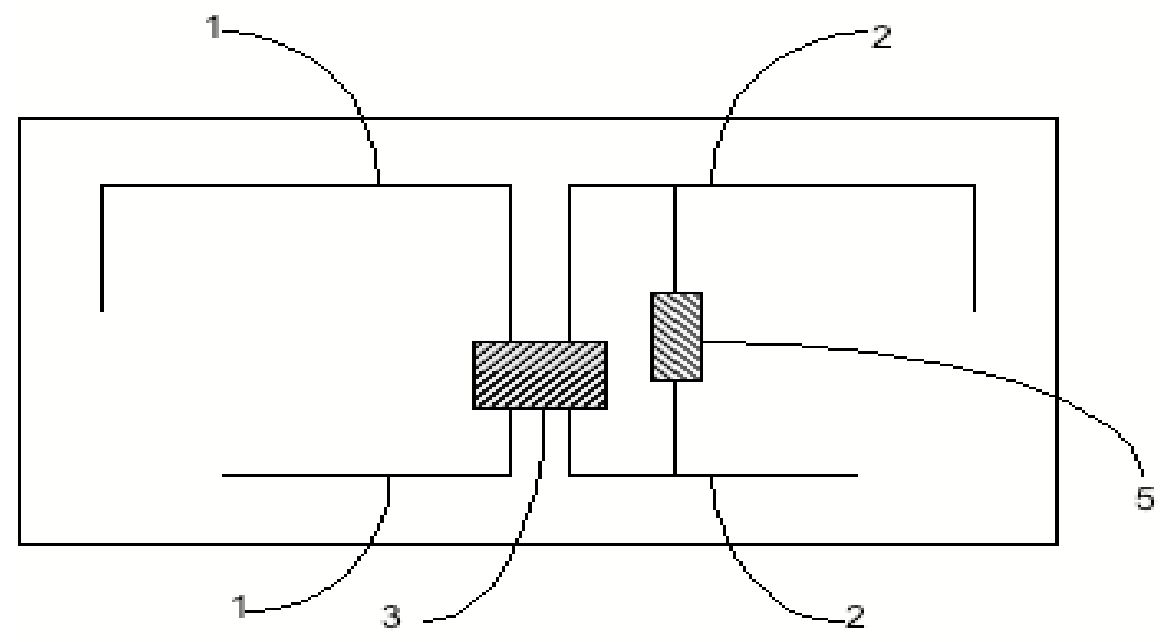

Fig. (5). Sketch figure of 'non-lead pin' structure.

this instance, the antenna linked to temperature sensitive components is working under the second resonance frequency.

\section{WORKING FLOW OF INTEGRATION DESIGN BETWEEN TEMPERATURE SENSITIVE COMPO- NENT AND RFID TAG}

The first resonance frequency and signal intensity of the dual-label design to measure temperature introduced above will keep fix when placed under a certain temperature level for a period of time. In this condition, the first RFID tag is working under the first resonance frequency. Temperature change outside will make temperature sensitive component's resistance change. In this condition, at least one of the character frequency and signal intensity of the RFID tag will change when placed under a certain temperature level for a period of time, and the second RFID tag is working under the second resonance frequency.

Working flow of dual-label RFID to measure temperature is depicted as Fig. (6). Firstly, The first resonance frequency and signal intensity of the RFID tag introduced above will keep fix when placed under a certain temperature level for a period of time. In this condition, the first RFID tag is working under the first resonance frequency. Secondly, at least one of the character frequency and signal intensity of the RFID tag will change when placed under a certain temperature level for a period of time, and the second RFID tag is working under the second resonance frequency. Next, Commands are sent to the first RFID tag and the second RFID tag through reading instrument mentioned above, and meanwhile feedback signals are received. RFID reading instrument make the signal intensity value as a reference value from the first RFID tag, which does not link to temperature sensitive components, and make a compare to signal intensity from the second RFID tag, which link to temperature sensitive components. Finally, the change of temperature is checked and measured by comparing the difference between signal intensities of different frequency from the first RFID tag and the second RFID tag when they work.

Actually, the purpose to deploy this RFID reading instrument is to transform the received antenna signal intensity value into deferent environment temperature value by using stored reference data. Reading instrument could send a command between channel n-1 and channel $n$ to further con- 
The first resonance frequency and signal intensity of the RFID tag introduced above will keep fix when placed under a certain temperature level for a period of time. In this condition, the first RFID tag is working under the first resonance frequency

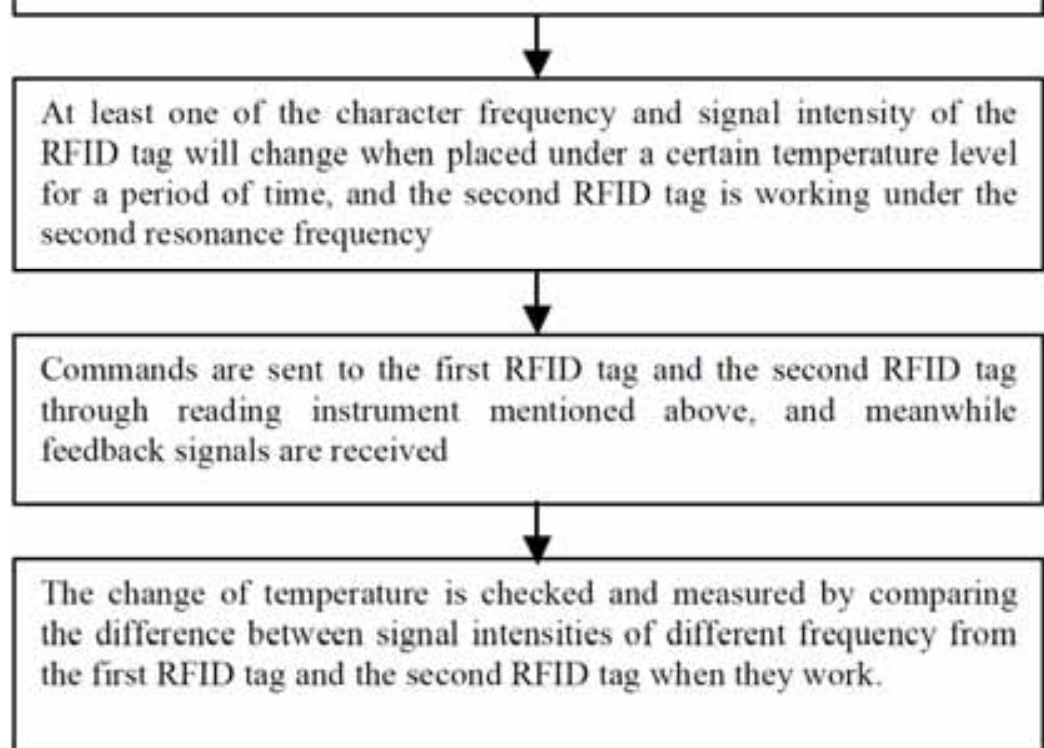

Fig. (6). Working flow of dual-label to measure temperature.

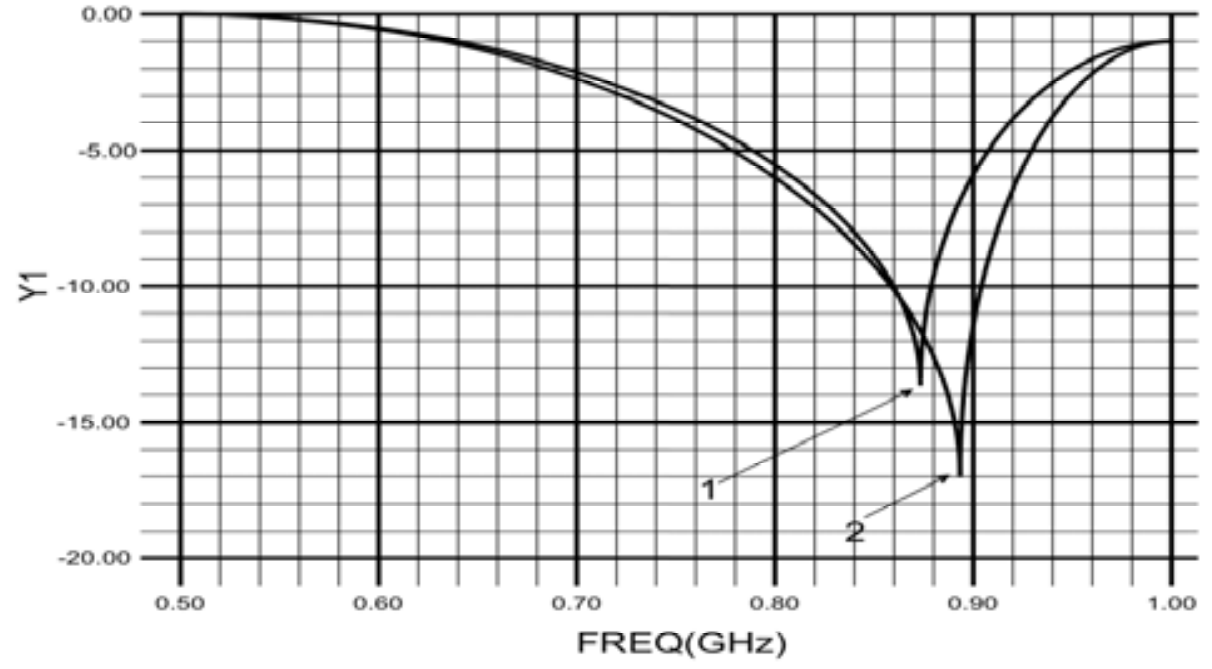

Fig. (7). Resonance frequency of RFID tag antenna under original temperature.

firm whether working frequency range of tag antenna has experienced excursion. Because tag could receive commands sent from channel $\mathrm{n}-1$, and feed back information through channel $\mathrm{n}-1$ but could not receive commands sent from channel $\mathrm{n}$, also could not feed back information through channel $\mathrm{n}$, therefore whether working frequency of tag antenna has experienced excursion could be confirmed in this way. Furthermore, the process of method or algorithmic depicted in our design could embed hardware, processor executive software, or integration of both directly. Software module could be stored in RAM memorizer, flash memorizer, ROM memorizer, EPROM memorizer, EEPROM memorizer, register, hardware, shiftable disk, CD-ROM or any other storage media in this domain.

\section{EXPERIMENT RESULT AND ANALYSIS}

An experiment is carried out according to the design scheme mentioned above, the result is depicted as Fig. (7) and Fig. (8). The difference value of resonance frequency of the first RFID tag and the second RFID tag changes as the environment temperature change. As depicted in Fig. (7), under original temperature, the resonance frequency 1 of the first RFID tag and the resonance frequency 2 of the second RFID tag almost keep consistent with each other and both are close to $0.90 \mathrm{GHZ}$.

As depicted in Fig. (8), when temperature change as time goes by, the resonance frequency of the second RFID tag decrease to about $0.70 \mathrm{GHZ}$, because the temperature sensi- 


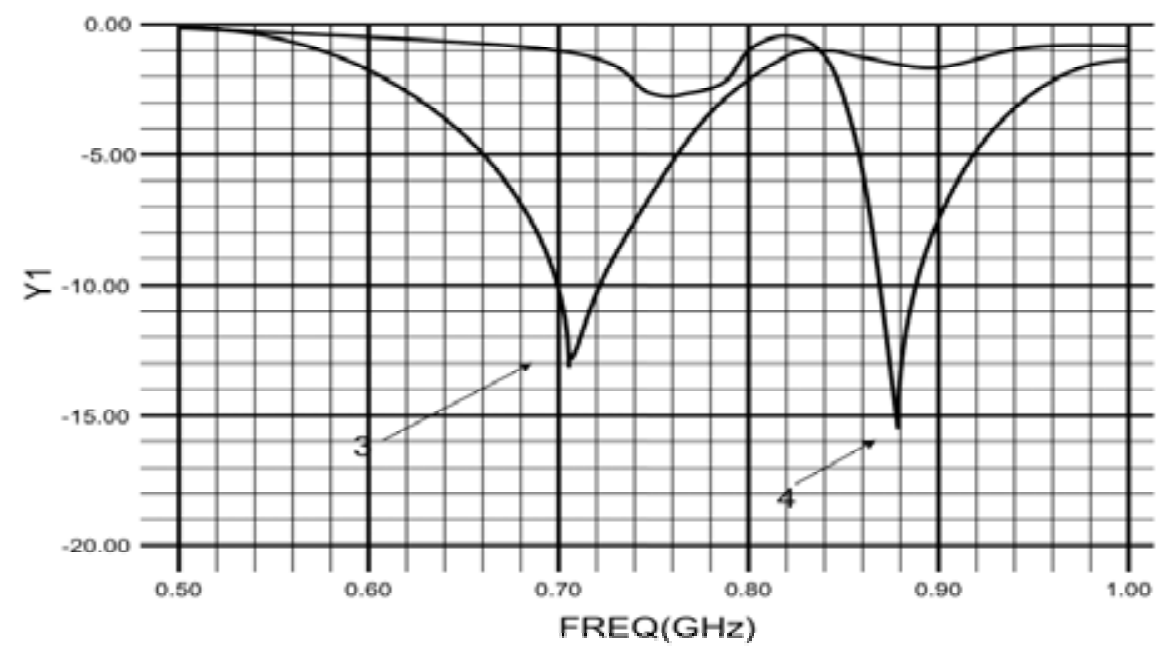

Fig. (8). Resonance frequency of RFID tag antenna under changed temperature.

tive component is deviced in antenna. However, the resonance frequency of the first RFID tag that does not link to temperature sensitive component is still about $0.90 \mathrm{GHZ}$. The inherent relationship between environment temperature and resonance frequency difference could be arrived by registering character frequency and signal intensity of the second RFID tag with temperature sensitive component and comparing with the second RFID tag which doesn't have temperature sensitive component. Thereby the function of measuring temperature could be realized by using RFID tag with temperature sensitive component.

\section{CURRENT AND FUTURE DEVELOPMENTS}

This paper proposes a new type of design scheme, which is used to solve the sensor and RFID tag integration problem by summarizing present studies and deeply analyzing correlated technologies. The design scheme is to extract the core part (temperature sensitive component) of temperature sensor, and integrate with RFID tag technology in an innovative form. This kind of design of dual-label to measure temperature meliorates the former technology system in some extent, and reduces producing cost comparing with present technology integration scheme. Moreover, the problem of deciding mark is solved in low cost in the process of using facility by alternate communicating with the first tag and the second tag, and making signals of the first tag antenna which doesn't link to temperature sensitive component as a reference value. Meanwhile, the change caused by coupling between tag and reading instrument could be filtrated, which makes the measure result even more precise. In addition, the problem of power supply in the process of using facility is solved by using tag's antenna and radio frequency module to transform electromagnetic wave into electric energy, which could supply power to RFID tag and temperature sensitive components. Whereas, in this design, data might be interfered in the process of transmission. The circuitry between lead pin and temperature sensitive component also affects the precision of measurement. So in the future, the design needs to further improvement to adapt more precise measure requirement. Moreover, integration of different kinds of sensitive components and RFID tag still needs further study. In a word, Low cost, miniaturization, intelligence, environmental protection, standardization, high accuracy, versatility, high reliability and security are a direction for both temperature sensor and RFID tag of future developments. There's no doubt that their applications will be expanded widely in high-tech fields except for those traditional fields. Furthermore, the integration of RFID technology and sensor technology will develop more deeply and extensively with the constant development of the internet of things technology and information industry.

\section{CONFLICT OF INTEREST}

The authors confirm that this article content has no conflict of interest.

\section{ACKNOWLEDGEMENTS}

This work was supported by doctorial fund of hebei normal university of science and technology, 2010YB001.

\section{REFERENCES}

[1] W. Liu, Y. Zhuang, Z. Qi, and L. Tang, "Design of temperature sensor embedded in passive UHF RFID tag", J. Electron. Measure. Instrum., vol. 25, no. 5, pp. 468-473, May 2011.

[2] L. Li, S. Xie, and X. Huang, "A CMOS temperature sensor used in passive RFID tag”, Chinese. J. Sens. Actuat., vol. 23, no. 8, pp. 1098-1101, Aug. 2010.

[3] H. Meng, and H. Zhang, "Design of extreme low power temperature and humidity sensor tag based on active RFID", Microcomp. App., vol. 30, no. 5, pp. 20-23, Mar. 2011.

[4] B. Yan, “Active temperature tag”, China Patent 102269625A, Dec. 7, 2011.

[5] L. Kong, Y. Wu, L. Xiong, and Z. Wang, "Semi-active RFID temperature sensor electronic tag", China Patent 201927070U, Aug. $10,2011$.

[6] S. Wang, “Temperature sensor”, China Patent 202403827U, Aug. 29,2012 .

[7] H. Zhang, "Passive wireless temperature sensor", China Patent 103076105A, May 1, 2014.

[8] Z. Hou, "An intelligence sensor with RFID function and storing temperature", China Patent 201983880, Sept. 21, 2011.

[9] L. Sun, “Wireless sensor realized by RFID tag”, China Patent 202584175 U, Dec. 5, 2012. 
[10] L. Huo, "RFID label with thermosensitive device RFID system and temperature detection method", China Patent 102722746A, Oct.10, 2012.

[11] C. Timothy, S. Andreas, and B. Benjamin, "RFID Tag Device With Temperature Sensitive Antenna," U.S. Patent 2010/0079288A1, April 1, 2010.

[12] I. Forster, B. Roberts, "RFID device with patterned antenna, and method of making", U.S. Patent 20050200539 A1, Sept. 15, 2005.

[13] M. Kai, "Teruhisa Ninomiya,"Antenna sheet, tag, and method of manufacturing tag", U.S. Patent 20120013469 A1, Jan. 19, 2012.

[14] C. Zhang, X. Qiu, H. Xia, and T. Ma, "Technology and application of the internet of things", Beijing: People Post and Telecommunication Press, pp.1-4, Jan. 2011.
[15] Y. Zhang, and X. Hu, "The Application of sensor", Fujian: Fujian Science and Technology Press, pp.10-15, Jan. 2006.

[16] B. Guan, L. Zang, S. Zhang, B. Chen, and J Li, "RFID tag antenna simulation design method and tag antenna", China Patent 102709688A, Oct. 3, 2012.

[17] M. Kai, and T. Ninomiya, "Radio frequency identification tag and antenna", U.S. Patent 8276825B2, Oct. 14, 2012.

[18] T.P. Wang, T.C. Ni, S-Y. Wang, and H.H. Chou, "RFID antenna and electronic product having the RFID antenna", U.S. Patent 8009105B2, Aug. 31, 2011.

[19] J.Y. Choo, J.K. Hong, J.K. Ryoo, "Radio frequency identification antenna", European Patent 2348462 A1, July 27, 2011.

Received: June 09, 2014

Revised: June 22, 2014

Accepted: July 24, 2014

(C) Zhang et al.; Licensee Bentham Open.

This is an open access article licensed under the terms of the Creative Commons Attribution Non-Commercial License (http://creativecommons.org/licenses/by-nc/3.0/) which permits unrestricted, non-commercial use, distribution and reproduction in any medium, provided the work is properly cited. 